\title{
A GENERALIZED WEYR CHARACTERISTIC ${ }^{1}$
}

\author{
BY A. N. FELDZAMEN
}

Communicated by Einar Hille, November 29, 1958

The classical spectral multiplicity theory for a single normal operator on Hilbert space generalizes the unitary determination of a normal matrix by the multiplicities of its eigenvalues. We outline here the beginnings of an analogous equivalence theory patterned after the similarity determination of an arbitrary (complex) matrix by the Jordan canonical form, or the numerical invariants, the Weyr and Segre characteristics. Generalizations of these characteristics are defined, in spatial rather than combinatorial terms, in the Banach space context, but for application to single operators both abstract structural knowledge of the operators and a multiplicity theory of the classical kind are required; hence our equivalence conclusions are restricted to a class of spectral operators on Hilbert space. Here, the generalized Weyr characteristic provides a complete set of invariants for an equivalence relation slightly more general than similarity. No separability assumptions are required.

We assume various parts of spectral operator (and related) theory outlined in $\$ 2$ of Dunford's review article [2], including the multiplicity theory of Bade (cf. pp. 235-236 of [2]) for a complete Boolean algebra of projections on a Banach space, and also, the multiplicity theory in toto of Halmos [4] for a single normal operator on Hilbert space.

1. Let $\mathfrak{X}$ be a Banach space of uniform multiplicity $n<\infty$ with respect to the complete countably-additive spectral measure $E(\cdot)$, defined on the Borel sets $\mathfrak{B}$ of the complex plane $\mathcal{C}$, with support $\Lambda \in \mathfrak{B}$. (That is, if for $x \in \mathfrak{X}, \mathfrak{M}(x)$ denotes $\operatorname{clm}[E(\delta) x \mid \delta \in \mathfrak{B}]$, then, whenever $E(\delta) \neq 0$, the space $E(\delta) \mathfrak{X}=\bigvee_{i=1}^{n} \mathfrak{M}\left(x_{i}\right)$ and $n$ vectors are always required.) If $f$ is a Borel function on $\mathfrak{C}$ we write $S(f)$ for the not-necessarily-bounded operator $\int f(\lambda) E(d \lambda)$, and call a set $\delta \in \mathfrak{B}$ an inverting set for $S(f)$ if $E(\delta) S(1 / f)$ is bounded. Let

$$
C(x)=\Lambda\{E(\delta) \mid E(\delta) x=x, \delta \in \mathfrak{B}\}
$$

$-C(x)$ is always some $E(\delta)$-and call two sets $\delta, \pi \in \mathfrak{B}$ equivalent

1 This paper summarizes the author's doctoral dissertation at Yale University, written under the direction of Professor Nelson Dunford. The work was supported in part by National Science Foundation Grant 3463. 
$(\delta \equiv \pi)$ if $E(\delta)=E(\pi)$, and a set $\delta \in \mathfrak{B}$ negligible if $\delta \equiv \varnothing$, the null set. We note that Bade has shown that

$$
\mathfrak{M}(x)=\{S(f) x \mid x \in \text { domain } S(f)\},
$$

and, if $\mathfrak{X}=\bigvee_{i=1}^{n} \mathfrak{M}\left(x_{i}\right)$, then

$$
\mathfrak{M}\left(x_{j}\right) \wedge\left(\bigvee_{i \neq j} \mathfrak{M}\left(x_{i}\right)\right)=0
$$

for each $j$ and every $x \in \mathfrak{X}$ can be written $x=\lim _{k \rightarrow \infty} \sum_{i=1}^{n} E\left(\pi_{k}\right) S\left(f_{i}\right) x_{i}$, where $\pi_{k}=\left\{\lambda|| f_{i}(\lambda) \mid \leqq k, i=1,2, \cdots, n\right\}, k=1,2, \cdots$.

A finite family of vectors $\left\{y_{1}, \cdots, y_{m}\right\} \subset \mathfrak{X}$ will be called independent if there is a family $\left\{z_{1}, \cdots, z_{m}\right\} \subset \mathfrak{X}$ satisfying (a) $\mathfrak{M}\left(y_{i}\right) \subset \mathfrak{M}\left(z_{i}\right)$, each $i$; (b) $C\left(z_{i}\right)=I$, each $i$; and (c) $\sum_{i} S\left(f_{i}\right) z_{i}=0$ implies each $S\left(f_{i}\right) z_{i}=0$, for every family $\left\{f_{1}, \cdots, f_{m}\right\}$ of Borel functions with $z_{i} \in$ domain $S\left(f_{i}\right)$. Then the standard elementary arguments for finite-dimensional vector spaces can be modified, using inverting sets whenever the invertibility of a scalar is required, to prove the following theorem.

THEOREM 1. The maximum cardinality of an independent family of vectors in $\mathfrak{X}$ is $n$.

If $\mathfrak{X}=\bigvee_{i=1}^{n} \mathfrak{M}\left(x_{i}\right)$ then $\left\{x_{1}, \cdots, x_{n}\right\}$ is independent. An obvious decomposition of $\Lambda$ can be used to prove Theorem 2 .

Theorem 2. The family $\left\{y_{1}, \cdots, y_{m}\right\} \subset \mathfrak{X}$ is independent if $\Lambda_{i} C\left(y_{i}\right) \neq 0$ and $\sum_{i} S\left(f_{i}\right) y_{i}=0$ implies each $S\left(f_{i}\right) y_{i}=0$ for every family of Borel functions $\left\{f_{1}, \cdots, f_{m}\right\}$ with $y_{i} \in \operatorname{domain} S\left(f_{i}\right)$.

Now let $Q$ be a fixed quasi-nilpotent operator commuting with $E$. We desire to prove the nonzero orbit of every $x \in \mathfrak{X}$ under $Q$, $\left\{x, Q x, Q^{2} x, \cdots\right\}$, is independent. The next theorem follows directly from the commutativity.

Theorem 3. For every $x \in \mathfrak{X}, C\left(Q^{k} x\right) \geqq C\left(Q^{k+1} x\right), k=0,1,2, \cdots$.

Theorem 4. If $Q x=S(f) x$, then $Q x=0$.

Only for this theorem is the quasi-nilpotency required, and this can be weakened. We sketch a proof. Assume $Q x=S(f) x \neq 0$. Then there is a set $\delta \in \mathfrak{B}$ such that $|f(\lambda)|>\epsilon>0$ for $\lambda \in \delta$, and $E(\delta) x \neq 0$. It is clear that $Q^{k} x=S\left(f^{k}\right) x$. Then, in Hilbert space,

$$
\left|Q^{k}\right|^{2}|E(\delta) x|^{2} \geqq\left|Q^{k} E(\delta) x\right|^{2}=\left|\int_{\delta} f^{k}(\lambda) E(d \lambda) x\right|^{2}>\epsilon^{2 k}|E(\delta) x|^{2},
$$

contradicting the quasi-nilpotency of $Q$. The proof in Banach space is 
similar, using a polar decomposition of $f$ and a Bade functional (cf. Theorem 3.1 of [1]) in place of the inner product.

Theorem 5. If $Q^{i} x \in$ domain $S\left(f_{i}\right)$ and $\sum_{i=0}^{m} S\left(f_{i}\right) Q^{i} x=0$, then each $S\left(f_{i}\right) Q^{i} x=0$.

If not, we can suppose, using inverting sets, that $S\left(f_{m}\right)=I, Q^{m} x \neq 0$, and each $S\left(f_{i}\right)$ is bounded. Let $\prod_{i=1}^{m}\left[t-g_{i}(\lambda)\right]$, where each $g_{i}$ is a bounded Borel function, be a factorization of the polynomial $\sum_{i=0}^{m} f_{i}(\lambda) t^{i}, f_{m}(\lambda)=1$ (cf. Lemma 3.1 of $\left.[3]\right)$. Let $z_{1}=\prod_{i=2}^{m}\left[Q-S\left(g_{i}\right)\right] x$. Then $\left(Q-S\left(g_{1}\right)\right) z_{1}=0$, so $Q z_{1}=\prod_{i=2}^{m}\left[Q-S\left(g_{i}\right)\right] Q x=0$. Let $z_{2}$ $=\prod_{i=3}^{m}\left[Q-S\left(g_{i}\right)\right] Q x ;$ similarly $Q z_{2}=\prod_{i=3}^{m}\left[Q-S\left(g_{i}\right)\right] Q^{2} x=0$. Repetition shows $Q^{m} x=0$.

CoRollary. If $x \in \mathfrak{X}$ then the nonzero orbit of $x$ under $Q$ is independent. Thus $Q^{n} \mathfrak{X}=0$.

If $\varnothing \neq \equiv \delta \in \mathfrak{B}$, we call a (finite) family of vectors $\left\{x_{\alpha} \mid \alpha \in A\right\}$ a $k$ th index system over $\delta$ if (a) $Q^{k} x_{\alpha}=0, \alpha \in A$; (b) $C\left(Q^{k-1} x_{\alpha}\right) \geqq E(\delta), \alpha \in A$; and (c) the family $\left\{Q^{i} x_{\alpha} \mid \alpha \in A, i=0,1, \cdots, k-1\right\}$ is independent.

Theorem 6. If $\phi \neq \equiv \in \in \mathfrak{B}$, the cardinality of a maximal kth index system over $\delta$ is unique.

Let $\mathcal{W}(\delta, k)$ be this cardinality (and be zero when $\delta \equiv \varnothing)$. Then $W(\delta, k) \leqq n$ for each $k$, and $W(\delta, k)=0$ for $k>n$.

Theorem 7. (a) For every $\delta \in \mathfrak{F}, \mathfrak{W}(\delta, k) \geqq W(\delta, k+1)$ for each $k=1,2, \cdots$.

(b) If $\pi \in \mathfrak{B}, \varnothing \neq \equiv \subset \delta$, then $W(\pi, k) \geqq W(\delta, k)$ for each $k$.

(c) If $\delta$ is the disjoint union of a countable family $\left\{\delta_{i}\right\} \subset \mathfrak{B}$ of nonnegligible sets, then $\left.\mathscr{W}(\delta, k)=\min _{i}\left\{W_{(}, k\right)\right\}$ for each $k$.

Let $\delta(\delta, k)=\mathscr{W}(\delta, k)-\mathscr{W}(\delta, k+1)$. Then $\sum_{k=1}^{\infty} k S(\delta, k)$ $=\sum_{k=1}^{\infty} \mathscr{W}(\delta, k)$, and $\mathscr{W}$ and $\delta$ are the Weyr and Segre characteristics in the finite dimensional case when $\delta=\{\lambda\}$ is an eigenvalue. Theorem 7 shows $\mathscr{W}(\cdot, k)$ to be a multiplicity function in the sense of Halmos for each $k$. Call a set $\delta \in \mathfrak{B} k$-uniform if $\varnothing \neq \equiv \pi \subset \delta$ implies $\mathscr{W}(\pi, k)$ $=\mathscr{W}(\delta, k)$, and of uniform characteristic if it is $k$-uniform for every $k$. It then follows from the arguments of [4] that $\Lambda$ can be partitioned into sets of uniform characteristic.

Theorem 8. For each $\delta \in \mathfrak{B}, \sum_{k=1}^{\infty} \mathscr{W}(\delta, k) \leqq n$.

Our chief result in the Banach space context is Theorem 9, required for the equivalence theory of the next section. 
Theorem 9. A non-negligible set $\delta \in \mathfrak{B}$ has uniform characteristic if and only if $\sum_{k=1}^{\infty} \mathscr{A}(\delta, k)=n$.

The obvious correspondence between equivalence classes of Borel subsets of $\Lambda$ and equivalence classes (absolute continuity) of regular, totally-finite, countably-additive, non-negative set functions (=measures) with support in $\Lambda$ permits us to consider $W$ defined for measure, rather than set, arguments.

2. Abandon the assumption of uniform multiplicity and take the underlying vector space to be an arbitrary Hilbert space $\mathfrak{S}$. Let $N$ be a (bounded) normal operator on $\mathfrak{S}$ with resolution of the identity $E$ and multiplicity function $u$, from measures to cardinals, characterizing $N$ to within unitary equivalence. We recall there is a mapping $\mu \rightarrow C(\mu)$ of measures to self-adjoint projections in the second commutator (=weak or strong algebraic closure $\supset$ completion) of $E$, and a (nonunique) orthogonal family of measures $\left\{\mu_{\alpha} \mid \alpha \in A\right\}$ of uniform multiplicity with $\mathrm{V}_{\alpha} C\left(\mu_{\alpha}\right)=I$. Each subspace $C\left(\mu_{\alpha}\right) \mathfrak{E}$ is a space of uniform multiplicity $u\left(\mu_{\alpha}\right)$ with respect to the countably decomposable (hence complete) spectral measure $C\left(\mu_{\alpha}\right) E(\cdot)$. We call $N$ essentially finite if $u(\mu)$ is always finite. If $Q$ is a quasi-nilpotent commuting with $N$ then $Q$ is completely reduced by each $C\left(\mu_{\alpha}\right) \mathfrak{W}$, and, if $N$ is essentially finite, the results of $\S 1$ permit us to define $\varpi(\mu, k)$ for a measure $\mu$ satisfying $\mu \ll \mu_{\alpha_{0}}$ for some $\alpha_{0} \in A$. Then, for any measure $\mu$, let $\mathscr{W}(\mu, k)=\min _{\alpha}\left\{\mathscr{W}\left(\mu \wedge \mu_{\alpha}, k\right) \mid \mu \wedge \mu_{\alpha} \neq 0\right\}$. This definition is independent of the particular family $\left\{\mu_{\alpha}\right\}$-thus $W$ depends only on the pair $\langle N, Q\rangle$-and the significant results of $\$ 1$ (Theorems $7,8,9)$ mutatis mutandis follow readily.

THEOREM 10. If $S_{1}$ and $S_{2}$ are scalar operators on $\mathfrak{S}$ with respective resolutions of the identity $E_{1}$ and $E_{2}$ and $L$ is a nonsingular operator (i.e., bounded and with bounded, everywhere defined inverse) on $\mathfrak{W}$ such that $S_{2}=L S_{1} L^{-1}$, then $E_{2}(\cdot)=L E_{1}(\cdot) L^{-1}$. If $S_{1}$ and $S_{2}$ are normal as well, then they are unitarily equivalent.

Every scalar operator $S$ on $\mathfrak{S}$ has a normal conjugate, $L S L^{-1}, L$ nonsingular. Call $S$ essentially finite if one (and, by Theorem 10 , every) normal conjugate is essentially finite, and the spectral operator $T=S+Q$ (in canonical decomposition) essentially finite when $S$ is.

Theorem 11. Let $T_{1}=N_{1}+Q_{1}$ and $T_{2}=N_{2}+Q_{2}$ be similar (i.e., $T_{2}$ $=L T_{1} L^{-1}, L$ nonsingular) spectral operators on $\mathfrak{S}$ in canonical decomposition with normal scalar parts. If $T_{1}$ is essentially finite, so is 
$T_{2}$, and the Weyr characteristics defined by $\left\langle N_{1}, Q_{1}\right\rangle$ and $\left\langle N_{2}, Q_{2}\right\rangle$ are the same.

Thus if $T=S+Q$ is an essentially finite spectral operator we can unambiguously define its Weyr characteristic as that of any pair $\left\langle L S L^{-1}, L Q L^{-1}\right\rangle, L S L^{-1}$ normal, and it is a similarity invariant. Unfortunately, simple infinite matrix examples will show it is not a complete set of similarity invariants, and some form of unbounded similarity that will generate an equivalence relation is required. We call two spectral operators $T_{1}$ and $T_{2}$ semi-similar if there is, for $i=1,2$, a family of projections $\left\{P_{\alpha}^{i} \mid \alpha \in A\right\} \subset \bar{E}_{i}$ (the completion of the resolution of the identity $E_{i}$ of $T_{i}$ ) with $\bigvee_{\alpha \in A} P_{\alpha}^{i}=I$, such that, for each $\alpha \in A$, there is a nonsingular operator $L_{\alpha}$ from $P_{\alpha}^{1} \mathfrak{S}$ onto $P_{\alpha}^{2} \mathfrak{S}$ with $T_{2} P_{\alpha}^{2}=L_{\alpha} T_{1} L_{\alpha}^{-1} P_{\alpha}^{2}$. (As $P_{\alpha}^{i} \in \bar{E}_{i}$, the operator $T_{i}$ and its scalar and quasi-nilpotent parts are completely reduced by each $P_{\alpha}^{i} \mathfrak{S}$, and $T_{1} P_{\alpha}^{1}=L_{\alpha}^{-1} T_{2} L_{\alpha} P_{\alpha}^{1}$.)

Semi-similarity is an equivalence relation for spectral operators, semi-similar normal operators are unitarily equivalent, and the spectrum is a semi-similarity invariant. If $T_{1}$ and $T_{2}$ are semi-similar spectral operators and $T_{1}$ is essentially finite, then $T_{2}$ is essentially finite. The Weyr characteristic is a complete set of semi-similarity invariants for essentially finite spectral operators on $\mathfrak{S}$. That is,

THEOREM 12. Tro essentially finite spectral operators on $\mathfrak{S}$ are semisimilar if and only if they have the same Weyr characteristic.

\section{REFERENCES}

1. W. G. Bade, On Boolean algebras of projections and algebras of operators, Trans. Amer. Math. Soc. vol. 80 (1955) pp. 345-360.

2. N. Dunford, A survey of the theory of spectral operators, Bull. Amer. Math. Soc. vol. 64 (1958) pp. 217-274.

3. S. R. Foguel, Normal operators of finite multiplicity, Comm. Pure Appl. Math. vol. 11 (1958) pp. 297-313.

4. P. R. Halmos, Introduction to Hilbert space and the theory of spectral multiplicity, Chelsea, New York, 1951.

YALE UNIVERSITY 